\title{
Tachon Syndrome: Rare Side Effect of Articular Injections of Corticosteroids. A Report of Two Cases
}

\author{
Sonia Rekik ${ }^{1}$ (D) Soumaya Boussaid ${ }^{1} \cdot$ Hedia Ben Abla $^{1} \cdot$ Ilhem Cheour $^{1} \cdot$ \\ Med Ben Amor ${ }^{2} \cdot$ Med Elleuch ${ }^{1}$
}

Published online: 24 November 2017

(c) The Author(s) 2017. This article is an open access publication

\begin{abstract}
Epidural or intra-articular injections of corticosteroids are an option for the treatment of several pain conditions but are not without adverse effects. Here, we discuss a rare systemic side effect of this therapy: Tachon syndrome. We report two cases, a 64-year-old woman and a 43-year-old man, who presented with Tachon syndrome after receiving, respectively, a shoulder and a lumbar injection of cortivazol $3.75 \mathrm{mg} / 1.5 \mathrm{ml}$ suspension for injection in pre-filled syringes. The indication for this therapy was, respectively, tendinopathy of the supraspinatus and a mechanical L5 lumbosciatica. A few minutes after receiving the injection, patients experienced acute low back pain, chest tightness, facial erythema and profuse sweating. All vital and biologic parameters were normal. In the first case, improvement was spontaneous and all symptoms resolved in $20 \mathrm{~min}$. The second patient remained under observation and received an intravenous 'physiological' infusion. Both patients recovered fully and returned home. A causal relationship between the corticosteroid injections and the patients' symptoms was very likely because of the acute clinical presentation and the rapid improvement in the patients' conditions and that no further signs indicating other serious complications developed.
\end{abstract}

Electronic supplementary material The online version of this article (https://doi.org/10.1007/s40800-017-0062-z) contains supplementary material, which is available to authorized users.

Sonia Rekik

rekik.sonia80@yahoo.fr

1 Rheumatology Department, La Rabta Hospital, 1007 Jabbari Street, Tunis, Tunisia

2 ENT Department, La Rabta Hospital, Tunis, Tunisia

\section{Key Points}

Practitioners performing local corticosteroid infiltrations should be aware of the potential for Tachon syndrome but must also always eliminate any other emergency.

Patients who experience Tachon syndrome should be reassured, despite the syndrome's spectacular symptoms.

Tachon syndrome appears to be rapidly reversible without any aftermath.

\section{Introduction}

Epidural and intra-articular corticosteroid injections are a widespread practice in rheumatology and are useful treatment options for patients with disabling peripheral joint or lumbar pain [1]. Although the serious complications described in the literature are fortunately infrequent, it is quite common for patients to develop minor adverse effects such as Tachon syndrome (TS).

Although rare, TS can lead to possible anxiety for both patient and clinician and, in extreme cases, legal consequences. However, we found no extensive reports or reviews of this complication in the literature. We considered a report of these cases justifiable to allow us to discuss the clinical presentation, diagnosis and management of this syndrome. 


\section{Case Reports}

\section{Case 1}

A 64-year-old woman with a medical history of osteoporosis treated with alendronate for 3 years had experienced pain in her right shoulder for 4 months. On examination, the Jobe test was positive and the internal rotation of the shoulder painful. X-rays showed right trochiter condensation, and ultrasound exam revealed tendinitis of the supraspinatus. After anti-inflammatory, analgesic, local physiotherapy and rehabilitative treatments failed, a local injection of cortivazol provided an excellent initial result for 5 months, with no immediate allergic reactions or incidents. The patient presented with a relapse 6 months later, in January 2017, with clinical examination showing intense pain and limitation of the right shoulder, as in the previous episode. Neurological examination of the upper limbs was normal; no limitation or pain of the cervical spine was observed. Radiograph of the shoulder showed no additional abnormalities since the first exam, in particular, no calcifications.

A second local shoulder infiltration was indicated. We used a cortivazol (Altim ${ }^{\circledR}$ ) $3.75 \mathrm{mg} / 1.5 \mathrm{ml}$ suspension for injection in a pre-filled syringe. According to the same modalities, the injection was performed after skin disinfection with alcoholic betadine, without use of xylocaine ${ }^{\circledR}$. There was neither blood test during pre-injection aspiration nor resistance during injection. The infiltration lasted 2; 3 min later, the patient experienced acute low back pain, chest tightness, an erythematous rash of the face and profuse sweating. Blood pressure was $105 / 66 \mathrm{mmHg}$ and heart pulse was 63 beats per min.

We rapidly stretched out the patient and elevated her lower limbs. Electrocardiograph (ECG) was normal. Symptoms spontaneously improved in about $20 \mathrm{~min}$, with total resolution of dorsolumbar and thoracic pain and face erythema and normalization of blood pressure. However, the patient remained in observation because she continued to experience fatigue for about $30 \mathrm{~min}$. Thereafter, she fully recovered.

\section{Case 2}

A 43-year-old man with type 1 diabetes mellitus receiving insulin therapy experienced mechanical right L5 lumbosciatica in December 2015. He improved after three epidural injections in March 2016. In February 2017, he presented with a new episode of lumbosciatica. On examination, the Schober index was at $1.5 \mathrm{~cm}$ and Lasègue's sign was positive at $40^{\circ}$ on the right side without sensory or motor deficits, perineal numbness or bladder dysfunction. Lumbar computed tomography (CT) identified conflicting L5-S1 disc herniation. After anti-inflammatory therapy and oral analgesics failed, we decided to perform epidural infiltration using cortivazol $3.75 \mathrm{mg} /$ $1.5 \mathrm{ml}$ suspension for injection in a pre-filled syringe. After premedication with two tablets of paracetamol $500 \mathrm{mg}$ and disinfection of the skin, the injection was performed with the customary precautions and lasted 3;2 min after the injection, the patient experienced sudden discomfort with sweating, chest and then strong lumbar pain, facial erythema and headache. Blood pressure and glycemia were both normal at, respectively, $120 / 75 \mathrm{mmHg}$ and $1.3 \mathrm{~g} / \mathrm{l}$. The patient did not experience relief from rapid glucose administration or elevation of limbs. Intravenous 'physiological' infusion was performed. Urgent ECG and troponin dosage were normal. After $15 \mathrm{~min}$, all symptoms except for the headache had resolved. Neurological examination was normal. After $4 \mathrm{~h}$, the patient was well and safe to return home.

\section{Discussion}

In rare instances, an injection of local corticosteroids results in unexpected reactions. Among them, some immediate systemic effects, including headaches, allergy erythematous rash, pruritus, anaphylactic shock, palpitations, seizures and-sometimes-psychiatric disorders have been reported [2-5].

TS is one of these side effects. This unusual event is described by patients as excruciating pain and a feeling of imminent death. It is also an unforgettable experience for the physician [1]. TS is named after the French rheumatologist Gérard Tachon, who first reported his personal experience of several cases. The true incidence of TS secondary to corticosteroid infiltration is difficult to establish as literature on the subject is lacking. Indeed, this side effect is probably underestimated, and much of the information comes from the very few published clinical cases. To the best of our knowledge, the single scientific study by Jean-Marie Berthelot, published in 2004, is the only one to have investigated TS [1]. The authors presented the results of a retrospective survey in which a questionnaire was sent to 500 French rheumatologists with the aim of studying TS. In total, 318 cases were reported by 92 rheumatologists. Thus, this survey suggests that TS occurs in 1 in 8000 infiltrations or 6.5 years of practice [1] and seems to be more common in women. The clinical presentation of TS is reminiscent of the clinical features observed in transfusion incompatibility reactions [6-8]. Indeed, both conditions have in common intense discomfort associated with incoercible chest, lumbar or dorsal pain; restlessness; nausea; skin flushing; and dyspnoea 
$[1,6]$. The exception is that transfusion incompatibility reactions usually occur with transient shock and more slowly than TS, whereas TS occurs within 1-5 min (78\%) following corticosteroid injection [1]. It should be noted that our patients also experienced symptoms 2 and $3 \mathrm{~min}$ after the onset of corticosteroid therapy.

Studies have found lumbar pain to be present in $84 \%$ of similar conditions and dorsal pain in $25 \%[1,9]$. TS can be preceded by immediate simple facial flushing in $64 \%$, which resolves in about 36-72 h [10]. These clinical features are comparable to those observed in our patients. Of note, symptoms can also be much more impressive, with more serious signs: anxiety in $87 \%$, shortness of breath in $64 \%$, diffuse sweating $(41 \%)$, chest pain $(36 \%)$ or uneasiness (29\%). Less frequently, TS includes transient symptoms such as cough (23\%), abdominal pain (20\%), hypertension $(15 \%)$, hypotension $(8 \%)$, paleness $(10 \%)$, diarrhoea (3\%), headache (3\%), nausea or intense asthenia [1]. No cases of asthma attack, angioedema or other neurological signs have been cited.

A diagnosis of TS is usually difficult to establish, and the condition must be distinguished from aortic dissection, particularly when associated with disturbed vital signs or persistent symptomatology [11].

In the cases we present, we concluded the patients' symptoms were likely to be related to TS because the timing between the procedure and symptoms was suggestive and we were unable to find an alternative causal explanation.

As for the infiltration sites, Berthelot et al. [1] mentioned that TS occurred most frequently (39\%) with lumbar epidural injections. Nevertheless, in various studies reporting complications after epidural infiltration, this side effect is either not explicitly labelled as TS or its symptoms are simply quoted by authors. Indeed, a recent retrospective study analysing 4265 epidural corticosteroid infiltrations in 1857 patients over a 7-year period recorded 103 minor complications, representing $2.4 \%$ of side effects; the most common problem was immediate increased lumbar pain $(1.1 \%)$ [12]. This rate was estimated at $0.4-5 \%$ in the study by Botwin et al. [13], who also cited other symptoms such as flush, abdominal pain and transient headaches.

Some physicians have experienced but not recognised the clinical signs of TS and have been embarrassed by this unexplained instantaneous increase in lower back pain and the other possible clinical signs, especially with injection into a non-spinal site. This was the case with our first patient, who experienced intense dorsolumbar pain immediately after shoulder infiltration with cortivazol, symptoms that we were not able to explain credibly.

In fact, TS can occur even where the infiltration site is not the spine. TS has been reported with intra-articular injections of the upper limb (30\%), mainly the shoulder; the lower limb (24\%), mostly in the heel; as well as other locations $(7 \%)$, such as paravertebral areas.

All insoluble corticosteroids available for intra-articular or epidural injection can cause TS, but the drug cited most often (67\%) was cortivazol [1-9].

Although the precise pathogenesis of TS remains unclear, several predominant hypotheses have emerged. The benign nature of this rare side effect might be the first explanation for the lack of studies on this topic [14]. Another explanation was its possible confusion with allergy. However, TS is unlikely to be an allergic reaction or linked to allergic history. Only $2 \%$ of TS cases developed urticaria [15]. Moreover, such suracute axial or thoracic pains are not observed in allergic reactions $[15,16]$. TS was also not related to the underlying disease or to preexisting vasomotor instability [1]. Other theories have been debated, mainly implicating an unexpected vascular leakage of the corticosteroid suspensions via breaching of a large vein during the injection [7]. The breach can be formed even if the aspiration of blood before or during the injection reveals nothing. The frequent occurrence of dyspnoea before suracute axial pain could be explained by the flowing of the suspension of insoluble corticosteroid crystals first within the lung and then within the kidney filters compared with the reactions sometimes noticed after acute haemolysis following incompatible blood transfusions $[7,8]$. This theory seems to be the most legitimate because TS seems to occur more frequently after epidural injections, an area rich in venous plexus [14]. However, the delay between injection and the first clinical signs (usually 5-15 min) strongly suggests that TS is not only due to direct injection of corticosteroids into a blood vessel.

Laredo et al. [17] recently explored the mechanisms by which epidural injection of corticosteroids caused cord infarction. Their work may have uncovered the mechanism of TS: using intravitreal microscopy of mouse cremaster muscles, the authors tested direct injection of three types of corticosteroid suspensions (prednisolone acetate, triamcinolone acetonide and methylprednisolone acetate) and uncovered direct and obvious evidence that these drugs induced quick aggregation of red blood cells (RBCs), of which $28 \%$ are changed into spiculated cells, and this led to a progressively reduced blood flow until total arrest in small vessels in less than 1 min (video). Accordingly, this transient aggregation of RBCs in the lungs, kidneys and subcutaneous capillaries following venous systemic diffusion of particulate steroids could explain the chest and back pain in TS. Conversely, in this same study, intra-arterial administration of the cortivazol suspension in mouse vessels neither changed microvascular blood flow nor provoked RBC agglutination. In addition, neither formation of macroaggregates of particulate steroids nor arterial spasm was observed with cortivazol [17]. 
Therefore, it is not possible to discriminate whether only one or several mechanisms are responsible for TS.

Whether the RBCs of patients experiencing this impressive reaction are more sensitive to particulate steroids than others has not yet been addressed, but the rarity of TS compared with unintentional injection into veins suggests this hypothesis should be tested.

Nevertheless, other authors believe that an acute reaction of the sympathetic neurological system might have been involved in many cases of TS [1]. This would account for other reported features of TS, such as paleness, sweating and transient disturbed blood pressure.

Finally, the outcome of TS seems excellent. It often resolves quickly-in $10 \mathrm{~min}$ - but can persist for up to $25 \pm 71 \mathrm{~min}$ [14]. Our two patients swiftly experienced a favourable outcome. Only four cases of hospitalization due to TS were mentioned in the literature [11]. However, TS can recur in subsequent injections in 14-60\% of cases, and the symptoms can be more severe.

\section{Conclusion}

Although rare and rapidly reversible, TS can be one of the immediate side effects of corticosteroid infiltrations, with an outcome that is most often favourable. Physicians performing local infiltrations should be mindful of this possibility. Indeed, its spectacular and sudden symptoms are very distressing for the patient. Physicians must be able to reassure patients and explain the transient and benign nature of the syndrome. However, this syndrome remains a diagnosis of elimination that should only be retained after conventional medico-surgical emergencies are excluded. Further studies are needed to fully discern further evidence for the mechanism of this simple but curious adverse event.

\section{Compliance with Ethical Standards}

Funding No sources of funding were received in support of this work.

Conflicts of interest Sonia Rekik, Soumaya Boussaid, Hedia Ben Abla, Ilhem Cheour, Med Ben Amor and Med Elleuch have no conflicts of interest relevant to the content of this article.

Ethics All patients provided written informed consent for publication of their case reports. A copy of the written consent may be requested for review from the corresponding author.

Open Access This article is distributed under the terms of the Creative Commons Attribution-NonCommercial 4.0 International
License (http://creativecommons.org/licenses/by-nc/4.0/), which permits any noncommercial use, distribution, and reproduction in any medium, provided you give appropriate credit to the original author(s) and the source, provide a link to the Creative Commons license, and indicate if changes were made.

\section{References}

1. Berthelot JM, Tortellier L, Guillot P, Prost A, Caumon JP, Glemarec J. Tachon's syndrome (suracute back and/or thoracic pain following local injections of corticosteroids). A report of 318 French cases. Jt Bone Spine. 2005;72(1):66-8.

2. Maillefert JF, Aho S, Huguenin MC, Chatard C, Peere T, Marquignon MF, et al. Systemic effects of epidural dexamethasone injections. Rev Rhum. 1995;62(6):429-32.

3. Abram SE, O'Connor TC. Complications associated with epidural steroid injections. Reg Anesth. 1996;21(2):149-62.

4. Daragon A, Vittecoq O, Le Loet X. Visual hallucinations induced by intra-articular injection of steroids. $\mathrm{J}$ Rheumatol. 1997;24(2):411.

5. Hunter A, Blyth TH. A risk-benefit assessment of intra-articular corticosteroids in rheumatic disorders. Drug Saf. 1999;21(5):353-65.

6. Webster BH. Clinical presentation of haemolytic transfusion reactions. Anaesth Intensive Care. 1980;8(2):115-9.

7. Ballas SK. Sickle cell aneamia: progress in pathogenesis and treatment. Drugs. 2002;62(8):1143-72.

8. Friedman HD, Noonan M. Acute Gram-negative urosepsis mimicking an acute hemolytic transfusion reaction. Transfusion. 1996;36(5):460-5.

9. Kumar N, Newman RJ. Complications of intra and periarticular steroid injections. Br J Gen Pract. 1999;49(443):465-6.

10. DeSio JM, Kahn CH, Warfield CA. Facial flushing and/or generalized erythema after epidural steroid injection. Anesth Analg. 1995;80(3):617-9.

11. Berthelot JM, Le Goff B, Maugars Y. Side effects of corticosteroid injections: what's new? Jt Bone Spine. 2013;80(4):363-7.

12. Mcgraft JM, Schaefer MP, Malkamaki DM. Incidence and characteristics of complications from epidural steroid injections. Pain Med. 2011;12(5):726-31.

13. Botwin KP, Gruber RD, Bouchlas CG, Torres-Ramos FM, Hanna A, Rittenberg J. Complications of fluoroscopically guided caudal epidural injections. Am J Phys Med Rehabil. 2001;80(6):416-24.

14. Hajjioui A, Nys A, Poiraudeau S, Revel M. An unusual complication of intra-articular injections of corticosteroids: Tachon syndrome. Two case reports. Ann Readapt Med Phys. 2007;50(9):721-3 (718-20).

15. Mace S, Vadas P, Pruzanski W. Anaphylactic shock induced by intraarticular injection of methylprednisolone acetate. J Rheumatol. 1997;24(6):1191-4.

16. Montoro J, Valero A, Serra-Baldrich E, Amat P, Lluch M, Malet A. Anaphylaxis to paramethasone with tolerance to other corticosteroids. Allergy. 2000;55(2):197-8.

17. Laredo J, Laemmel E, Vicaut E. Serious neurological events complicating epidural injections of glucocorticoid suspensions: evidence for a direct effect of some particulate steroids on red blood cells. RMD Open. 2016;2(2):e000320. 